\title{
La comunicación y sus aportes disciplinares para el abordaje de experiencias de violencia sexual
}

Communication and its disciplinary contributions for the approach of sexual violence experiences

\author{
Rosaura Barrios rocha_barrios@yahoo.com.ar \\ http://orcid.org/0000-0001-7846-4364 \\ Instituto de Investigaciones Sociales y Humanas; \\ Facultad de Humanidades y Ciencias Sociales; \\ Universidad Nacional de Misiones (Argentina)
}

\section{Resumen}

El presente artículo es un recorrido analítico que busca dar cuenta de los aportes que puede realizar una disciplina como la Comunicación al abordaje de experiencias que tiene como víctimas de abuso sexual a niños, niñas y adolescentes. Más allá de los avances que presenta este artículo de análisis de las condiciones de producción de estos relatos que dan cuenta de un daño ocasionado por algún miembro de la familia del niño/a, lo que busca es poner en tensión nuestra participación en equipos de trabajo interdisciplinares que se dedican al cuidado, acompañamiento, tratamiento e intervención judicial de estos casos. Desde la comunicación podemos realizar aportes específicos no sólo al estudio de la experiencia dolorosa sino también comprender las lógicas de todo este engranaje organizacional y encontrar las formas de ver cómo articular la producción científica con intervenciones de este 
tipo. Para este trabajo desde la comunicación buscamos comprender las lógicas, analizar a partir de herramientas adecuadas al contexto e intervenir respetando todos los cuidados estipulados tanto nacionales como internacionales: Convención de los Derechos del Niño y de la Niña, Lineamientos éticos para el abordaje científico del CONICET, normativas legales para la protección de niños y niñas, protocolo de abordaje institucional interno de la ONG, Código de Ética para psicólogos y psicólogas.

Estas líneas son una propuesta de análisis y comprensión de estos equipos y sus laberintos, buscamos nuestro lugar en estas tramas y cómo intervenir a partir de sus aportes y el diálogo disciplinar.

Palabras Clave: Comunicación; abuso sexual contra niños, niñas y adolescentes; aportes disciplinares.

\section{Abstract}

This article is an analytical journey that seeks to account for the contributions that can be made by a discipline such as Communication to the approach of experiences that have as victims of sexual abuse children and adolescents. Beyond the advances presented in this article analyzing the conditions of production of these stories that account for an injury caused by a member of the child's family, what they seek is to put our participation in work teams in tension interdisciplinary that are dedicated to the care, accompaniment, treatment and judicial intervention of these cases. From the communication we can make specific contributions not only to the study of the painful experience but also to understand the logics of all this organizational gear and find ways to see how to articulate and intervene favorably to the victims. For this work from the communication we seek to understand the logics, analyze from appropriate tools to the context and intervene respecting all the stipulated care both nationally and internationally. These lines are a proposal for analysis and understanding of these teams and their labyrinths, we seek our place in these plots and how to intervene from their contributions and dialogue discipline.

Keywords: Communication; sexual abuse against children and adolescents; disciplinaries aports. 
Esta propuesta se desprende de las reflexiones del trabajo de campo para mi tesis doctoral (Barrios, 2016) desarrollada en una ONG que realiza asistencia y detección de problemáticas que tienen que ver con el abuso sexual a niños, niñas y adolescentes. Si bien yo quería ver los contextos en los que eran enunciados los testimonios que daban cuenta de un abuso sexual u otras huellas de violencia sexual, a lo largo de esos tres años pudimos notar potencialidades de la disciplina en este complejo campo que no solo involucra a la salud sino a otros organismos encargados de velar por estos niños y niñas. El poder judicial cuando interviene, los/as peritos/as de parte, abogados/as y defensores/as, la ONG en asistencia y otras organizaciones que colaboran en el cuidado de esta población.

Si bien la experiencia aquí fue a través de mi participación en un equipo de trabajo que abordó los testimonios a partir de la cámara Gesell, la misma nos permitió reflexionar el accionar de investigadores/as en comunicación en el abordaje de experiencias que tienen que ver con un abuso sexual y/o violación y están en proceso judicial: ¿Qué aportes podemos hacer desde la comunicación a problemas que competen al campo de la salud? En cuanto a esto decir las terapeutas que se abocan a esta tarea son vistas como profesionales de la salud, sin afectar muchas veces al cuerpo como materia, ellas sostienen que es un problema que debe abordarse con una perspectiva de salud pública. ¿Qué disciplinas dialogan en la construcción de un objeto propio del campo de la salud? ¿Cómo dialogamos con otras disciplinas? ¿Cómo construimos un problema polifónico? ¿Qué aporta -en definitiva- una comunicadora a estas tramas? ¿Cuál es la particularidad de su mirada para su inclusión a equipos de trabajo que aborden la experiencia traumática?

Estas líneas dan cuenta del recorrido analítico que se logró para luego pensar estrategias de intervención en los equipos de asistencia, entonces en estas líneas el/la lector/a encontrará una articulación entre marcos teóricos que habilitaron las reflexiones y colaboraciones que se lograron a raíz de estas reflexiones. La malla que sostienen estos análisis fueron los estudios de género y sexualidad(es) y los estudios en comunicación, específicamente los estudios culturales. No para ahondar en ellos ahora sino para prestar coordenadas teóricasmetodológicas a los/as lectores/as.

Hacia allá vamos.

Tres puntos para empezar a pensar la incorporación de comunicadores/as a equipos especializados en violencia sexual contra niños, niñas y adolescentes:

Es primera la suposición sobre la imposibilidad de narrar recuerdos dolorosos que tengan que ver con la violencia sexual. No fueron pocas las voces que vaticinaron que "nadie cuenta" un abuso sexual o una violación, mucho menos se abren las puertas para alguien que hace 
investigación social. La pregunta por esos silencios en la trama narrativa, o esos quiebres que se presentan como lo no dicho es lo que buscamos en principio. ¿El quiebre en el continuum de la vida supone el quiebre narrativo de la vida también? ¿Cómo irrumpe la violencia sexual no sólo en la dimensión vivencial sino del relato también? Sin ánimos de disociar ambas dimensiones, veremos como estas preguntas nos acercarán a la propuesta de trabajo que presentamos al inicio: el trabajo de comunicadoras/es en equipos de trabajo interdisciplinares especializados en la violencia sexual.

En el silencio arranca nuestro recorrido.

\section{El silencio}

A los y las comunicadoras el campo de la salud nos suele interpelar de dos maneras, por un lado en la práctica de lo que se conoce por comunicación pública de la ciencia y, por el otro, hacer investigación social de temas que abordan (también) desde la salud. La propuesta de este trabajo intenta ubicar a las comunicadoras y comunicadores a la par de otros profesionales que trabajan en consultorio o a través de otros dispositivos para el abordaje de problemáticas que involucran al dolor y al trauma.

¿Qué rol tiene una comunicadora en equipos de trabajo que tienen que ver con el rescate y asistencia de víctimas de violencia sexual? Trabajos como éstos ¿contribuyen, mejoran, intervienen de manera favorable para las víctimas? ¿Cómo poner este bagaje y accionar reflexivo al servicio de las organizaciones que trabajan en la búsqueda de justicia y reparación? ¿Podría existir un uso instrumental de las conclusiones del análisis sobre la experiencia narrativa (Barrios, 2016) (traumática) en situaciones de resolución concreta? ¿Cómo nos interpela ese dolor?

Para iniciar estas reflexiones traigo a colación una pequeña anécdota que tuvo lugar en la Universidad Nacional de Misiones en el marco de un Encuentro Regional de Antropo-semiótica de la muerte y el morir en la que fui invitada a discutir algunas de estas cuestiones que presento aquí. En ocasión de este panel discutíamos a cuatro años del femicidio de Lucía Maidana (1) y la pertinencia de las universidades públicas en los pedidos de justicia y su intervención:

Iván Bondar -Doctor, investigador del CONICET especialista en estudios que tienen que ver con la antropo-semiótica de la muerte y el morir- me responde: 
“¿Sabés por qué es legítimo trabajar con el dolor ajeno? Porque como investigadores habilitamos espacios para que se descarguen y puedan hablar de aquella persona que perdieron. Durante las entrevistas que duran horas pueden contar lo que atravesaron y desahogarse".

A esta intervención, la Mgter. Elena Maidana (2) responde:

"Eso desde el punto de vista de un metodólogo, nosotras en comunicación preferimos hablar de la habilitación de espacios de charla que permitan terminar de cerrar o terminar de vivir la experiencia... se traduce en escucha en nosotras pero habilita la palabra al otro."

Mi pregunta inicial fue ¿Es legítimo investigar narrativas que tienen que ver con el dolor del otro? Durante toda la discusión corrimos los límites y las preguntas alrededor de "lo que podemos o no" realizar en ciencias sociales, ¿hasta dónde podemos preguntar cuando investigamos?

Este intercambio en términos metodológicos que se dio en este panel fue la puerta para empezar a responder (o repreguntar) sobre la legitimidad de estudios sociales que involucren la pregunta sobre el dolor de un otro. ¿Qué objetivos debiéramos perseguir para contribuir al abordaje social de los mismos? Las conclusiones de este estudio (Autor, 2016) arrojó la posibilidad y la veta para comunicadores y comunicadoras interesados en estas problemáticas, una posibilidad de trabajar con otras organizaciones en el estudio y análisis de relatos sobre experiencia dolorosa e intervenir. Además de la psicología y el trabajo social otras disciplinas de igual forma pueden indagar, ¿qué pasaría si fuéramos incluidos en equipos de interdisciplina legal y forense para la preparación de pericias y contra pericias? Siempre nos han acusado a las ciencias sociales de no poseer una "aplicabilidad" instrumental de nuestros proyectos, de nuestros saberes, y aquí estamos ante un caso concreto de trabajo en campo en cruce con el trabajo legal.

¿Puede la comunicación, como disciplina, contribuir metodológicamente al estudio de problemas sociales? ¿Podemos lograr una ciencia más sensible, comprometida, cercana a sus pueblos? La agenda de investigación es un aporte para la construcción de puentes con la comunidad y pensarla desde otro lugar más allá de la figura de "informante" o testigo. Nacimos dialogando y todo indica que vamos hacia su consolidación como abordaje epistémicometodológico, es hora de encontrar la veta para fortalecer estas organizaciones y contribuir a su funcionamiento no sólo en un plano legal, sino en el armado de protocolos de intervención, de funcionamiento y de estudios sobre la violencia sexual.

Como pronosticaron al inicio de esta investigación hay silencios sí, pero en esos silencios hay palabras potenciales, hay diálogos y articulaciones, hay vericuetos. $Y$ en esos vericuetos nos 
metimos, sin saber que algunas respuestas estaban precisamente allí... en esos lugares que se mostraban como ocultos, silenciosos y silenciados.

Afortunadamente, para nosotras y nosotros desde la comunicación, el silencio comunica desde siempre. Y por él fuimos.

\section{De los espacios para contar y legitimar el dolor}

Desde la comunicación uno de los aportes que podemos realizar es la construcción y constitución de los lugares que buscan facilitar la enunciación de la experiencia dolorosa. Pero cuando abordamos estos problemas desde las ciencias sociales, ¿dónde está el límite de lo ético, de lo político y lo epistémico-metodológico? Allí donde hablamos del dolor del otro, donde la escucha efectiva se traduce en la posterior producción de cualquier documento público que dé cuenta de ese dolor, en este caso, las intervenciones judiciales en caso de abuso sexual a niños y niñas y adolescentes: ¿Cuáles son las condiciones de enunciación de estos testimonios? La creación de un espacio para la escucha de las víctimas no necesariamente viene a salvar estas tensiones éticas y políticas que sobrevuelan la producción del testimonio pero el análisis de las lógicas testimoniales son insumo para pensar protocolos de registro y sistematización. Por ejemplo, previo a la implementación de la cámara gesell en el país los niños y niñas debían testimoniar frente al juez, las modificaciones del Código Penal y sobre todo el artículo 221 bis que incorpora el uso de cámara gesell para menores de 18 años, reconoció con estas modificaciones la incapacidad de los miembros del tribunal para tomar declaraciones al niño o niña sin que sea revictimizado. Reconoce y visibiliza el accionar y el saber de otras disciplinas como la psicología para la toma de testimonio, muchas veces convertida en la única prueba con la que cuentan (¡miren sí será imprescindible profesionales específicos para la toma de este testimonio!). Aún así, y con la creación de estos (nuevos) espacios, las encrucijadas éticas y políticas no fueron saldadas, un/a comunicador/a entiende de formatos y géneros, identifica cuales son los adecuados para el contar teniendo en cuenta a sus interlocutores. Al ser el engranaje que articula todas estas disciplinas potencia cada aporte y lo calibra según las situaciones comunicativas.

Una de las maneras que posee el niño o niña para comunicar su dolor es a través de dibujos y juegos, elementos que son decodificados por la profesional de la salud. "¿Usted es la única que puede interpretar esos signos como indicadores de abuso?, preguntó en más de una 
ocasión un juez incrédulo. "¿Es posible que los miembros de este tribunal realicen la misma interpretación?" Siempre con un dejo de sospecha.

Aquí lo que ponen en cuestión, en realidad, es la propia legitimidad de una disciplina como la psicología para actuar en casos de abuso sexual a niños y niñas y su "real" contribución con sus materiales a la causa como prueba. Ponen sobre la mesa la trampa sectaria de la disciplina y su casi exclusividad para el trabajo con víctimas. ¿Qué costos tiene que todavía no hayamos podido avanzar como comunidad universitaria/académica en estos espacios donde una sola disciplina no puede dar batalla? No fueron pocas las veces en que el fiscal o el juez de parte pusieron en duda la capacidad, formación y experiencia de la psicóloga que en ese momento fue llamada a declarar o a presentar pericia en la causa, ellas no están protegidas por la ley que protege a los niños y niñas así es que no dudaron en ponerlas a declarar justo enfrente de los acusados.

Entonces, siempre el relato de la experiencia del niño/a estará mediado ya sea tecnológicamente a través del dispositivo de cámara gesell, a través de sus producciones didácticas- dentro del mismo espacio- y a través de un/a tercero/a que vendría a ser la terapeuta. Esto nos lleva necesariamente (como disciplina) a preguntarnos por las formas de presentar los relatos en estas instancias: ¿Es posible acceder a la experiencia dolorosa del niño o niña en primera persona sin tantas mediaciones (valga la redundancia) en el medio? ¿Es posible producir conocimiento científico sobre un otro? ¿Cómo salirse de cierta fascinación, estupor o indignación que causan los fenómenos de violencia para construir un "análisis razonado"? Lo anecdótico.

Desde sus inicios este trabajo fue pensado no sólo en la "soledad" de las reflexiones de un problema de investigación que deriva en un proyecto, sino siempre en diálogo y buscando la sintonía con su entorno. Este "en diálogo" (constante) significa: integrar como materiales de análisis todo registro que sea sensible de ser reflexionado y/o contribuya a la comprensión del problema de investigación. De estas reflexiones se desprenden fines instrumentales que buscan dar herramientas disciplinares a equipos de trabajo que se dedican a la búsqueda, investigación, reparación y acompañamiento de víctimas de violencia sexual en contextos de intervención de la justicia.

Como dijimos al inicio, suponíamos que íbamos a encontrar en estas travesías el silencio en su estado más doloroso. No estamos ante relatos pasados, quizás amortiguados con el pasar de los años, no menos dolorosos pero con el escudo de los años encima. Estamos ante potenciales relatos contados en primera persona de niños y niñas que están atravesando en este momento la experiencia del abuso sexual por parte de algún miembro de sus familias. Con 
el tiempo esos silencios se transformaron en "lo innombrable", aquello que no puede ser nombrado porque descoloca todas las categorías lingüísticas para dar cuenta de ese daño. ¿Podemos "soportar" no nominar, entender o nombrar aquello que nos aparece como desconocido al fin? ¿Cómo nombrar aquello que provoca una ruptura biográfica? Y para la confección de los materiales de análisis: ¿Cómo analizar aquello que no puede ser dicho?

Previo a estas intervenciones debieron hacerse los análisis y recorridos reflexivos. Desde la labor científica, siguiendo en esta línea de lo innombrable, lo intransferible, lo abyecto, lo desconocido, lo completamente otro... ¿De qué manera esa imposibilidad de nombrar nos lleva al descentramiento del análisis de los materiales de estudio? Ese descentramiento no solo desde la enunciación de la experiencia traumática sino (y en un grado de confusión no mayor, sí diferente) en la escucha científica de estos testimonios de experiencia dolorosa. Lo inenarrable, que resulta muchas veces ser el nudo de la narración de la experiencia traumática, aparece como obstáculo a nivel institucional en la resolución en un marco legal y un (si se quiere) llamado de atención sobre el estatuto de codificación o pasaje de formas de estos registros experienciales. Y es precisamente aquí donde está puesta la atención y vigilancia del investigador que busca analizar esas narrativas.

La preocupación es por el descentramiento del eje que trae la incapacidad de nominar/nombrar aquello que estamos percibiendo. Para la comunicación y el lenguaje, aquello que no se nombra, no existe. Pero allí está, se percibe al interior de la cámara gesell que hay algo que está provocando mucho dolor, mucho malestar y no está siendo codificado en palabras. Y la incertidumbre de no hallar categorías o colocarlas en los cajones adecuados provoca este descentramiento, es la extrañeza, la dificultad de no nombrar lo que desconcierta. "[...] nos invitan a reflexionar sobre la estrechez del discurso binario para nombrar(nos). 'Aquello que no se nombra, no existe' advierten y, a su vez, paradójicamente, 'nombrar es matar' señala Marla Morris en un texto compilado en castellano en Pensando queer. Sexualidad, cultura y educación de la editorial catalana Graó" (Pechín 2015, en Foro Debate "Género y Sexualidades"). Si bien Pechín se refiere a la "estrechez del discurso binario" para nominar a las identidades sexo-genéricas, nos valemos de esa punta para comprender los quiebres en el discurso y en las biografías que se encuentran huérfanas de parámetros lingüísticos.

Es en esta línea, y con esta misma preocupación, que Butler cuestiona a la misma norma para escapar a la antinomia.

"Quizás la más sugerente y sofisticada respuesta a este desafío la formuló hace unos años Judith Butler (1990), cuando en vez de quedarse dando vueltas alrededor de la tautología norma versus anti-norma (hetero versus homo; masculino versus femenino; homo versus trans, 
etc.) cuestionó la propia norma, su legalidad y su legitimidad como frontera que divide lo humano inteligible de lo no inteligible (y no humano), es decir, de lo abyecto." (Elizalde 2015, en Foro "Género y Sexualidades", Clase 1A).

Este desplazamiento que pone en limpio Elizalde citando a Butler es por demás tentadora: pasar de la "norma versus anti-norma" hacia la crítica a la propia norma, o hacia el mismo estatuto que permite la codificación o el pasaje de formas de la experiencia. Con el ojo puesto en Butler es posible realizar este ejercicio de reflexión: "El sujeto feminista resulta estar constituido discursivamente por el mismo sistema político que pretende facilitar su emancipación" (Butler, 1990: 2). En el relato de experiencia traumática en determinadas situaciones comunicativas la pregunta sería por el mismo relato de aquello que no puede ser nombrado, aquello que significó una ruptura en la subjetividad del sujeto, la misma debe ser pensada y codificada discursivamente por el mismo sistema que imposibilita su codificación o traducción a un relato institucional.

Con estas coordenadas es que abordamos metodológicamente los relatos de experiencias dolorosas que fueron enunciados institucionalmente. El recorrido metodológico trató de dar cuenta del mismo sistema que habilitó (y descartó) ciertos tipos de codificaciones de la experiencia, que fuera diagnosticada por las terapeutas como traumática.

El encuadre disciplinar que realizan las terapeutas a los testeos (3) no contribuye en gran medida para avanzar (satisfactoriamente) con la causa. Aún con los dibujos y testeos, aún con las Evaluaciones Psicodiagnósticas (EP) (4) los jueces siguen dictando la falta de pruebas:

¿Qué falla?: el abordaje y la construcción de esa veracidad. ¿Y si esa veracidad fuera construida en cooperación con otras disciplinas científicas? ¿Y si el abordaje de la EP fuera interdisciplinar? Porque la normativa ya dictó sus márgenes para la comprensión del delito pero algo está pasando que quedan restos, resabios sin enmarcar, sin contemplar. Pareciera que dentro de estas categorías la profundidad y enormidad del problema no caben. Estas marcas disciplinares no son suficientes, entonces ¿cómo puede contribuir la Comunicación Social como disciplina científica a la reflexión y acceso a la Experiencia Traumática? ¿Qué contribuciones disciplinares podemos prestar para su resolución judicial en términos favorables a las víctimas?

\section{¿Y la comunicación?}

Nuestro foco de atención para llegar a esta propuesta no fueron los relatos en sí, ni siquiera fue el acceso a la situación que provocó esa experiencia dolorosa. Nuestro foco de atención fue las 
condiciones que permitieron o facilitaron que esa experiencia dolorosa pueda ser enunciada y su posterior transformación en materiales legales/judiciales. De esto se deducen los aportes disciplinares de la comunicación.

¿Cómo dialogamos con la sicología, el trabajo social, la medicina forense? Y en este diálogo, ¿cómo construimos una mirada propia, plural, rigurosa y metodológica? La "salida" a estos laberintos la encontramos en lo metodológico, fue esa la veta que nos permitió dialogar y proponer sin perder especificidad y consistencia.

¿Es posible olvidar -y si no fuera así- es posible no contar? En un escenario donde pareciera que todo está a la vista, publicado, escrito, dicho, sentido, donde la denuncia y los procedimientos para llegar a ese relato son obligatorios ¿Qué tipo de resguardo tenemos con respecto a nuestros propios relatos, a nuestros propios recuerdos? $Y$ es que no sólo la enunciación tiene límites sino la propia representación de aquello que provocó tanto dolor, ¿hay lenguaje allí donde todo es dolor? La exposición a situaciones límites llevan también al límite la posibilidad misma de lo narrable (Aranguren Romero, 2010). Y frente a este contexto, ¿cómo interviene la comunicación como disciplina sin romper estos cuidados?

Cuando se realizó este trabajo de campo la escucha y la observación se convirtieron en herramientas fundamentales de acercamiento y sistematización. Adaptar esta investigación a los tiempos de las organizaciones se tradujo en estas herramientas sin registro de audio 0 escrito en el momento. La narración de experiencias de este tipo produce un quiebre en el lenguaje imposibilitando de que haya -efectivamente- relato. Todo es silencio, hueco, vacío. Situar las preguntas de un investigador en estos lugares significa imponer ritmos y formatos narrativos para el contar, por ende, rememorar, volver a vivir, supone intervenir en tiempos subjetivos de rememoración del horror y sufrimiento de la experiencia, busca gestionar lo indecible, de rellenar los huecos narrativos, de encontrar aquello inaprensible. No podía dejar (aun no) de ver la presencia de una investigadora en es(t)as tramas como intrusiva, es así que buscamos la forma de investigar con una metodología ajustada a estos cuidados. "Cuando se habla sobre violaciones, se le da una gran importancia a los silencios. Qué hacer con estos silencios -cómo escucharlos, cómo interpretarlos, cómo determinar cuando son opresivos y cuando pueden constituir una forma de agencia- es un tema de gran preocupación y debate." (Theidon, 2006: 71).

¿En qué medida las organizaciones que son facilitadoras de la enunciación de los relatos de estas víctimas permiten efectivamente la emisión de esta voz? ¿Qué géneros habilitan para moldear el sufrimiento y el dolor? ¿Puede narrarse el horror con éstos géneros disponibles 
para el contar? ¿En qué medida son compatibles estos géneros disponibles con los que necesita "la justicia" para proceder a favor de las víctimas?

Theidon, quien participó en las Comisiones por la Verdad y la Reconciliación en Perú (CVRP) en el 2003(5), trabaja con relatos enunciados en grupos focales, considera que el contexto de enunciación y recepción de estos testimonios son determinantes de las formas discursivas: "[...] me interesaban este tipo de recuerdos relatados en estos grupos focales porque el contexto en el cual los testimonios son dados y recibidos es central con relación a las formas que esos testimonios adquieren" (2006: 82). Esto y la constante pregunta por la ética en campo: ¿Tenemos derecho a preguntar sobre experiencias traumáticas? ¿Hay límites en investigación social? ¿Cuál es la metodología "menos" violenta e invasiva para el estudio de experiencias traumáticas? "No puedo separar los métodos de la ética: en este caso, ambos son repugnantes. Hay preguntas que no tenemos derecho a preguntar, y silencios que deben ser respetados" (Ídem: 87) Conscientes de que no podemos hacer investigación social en estas aguas sin tener estos recaudos de protección y cuidado de las víctimas pero incapaces de proponer formas de intervención disciplinaria sin investigación social tuvimos que hacer todas estas concesiones y negociaciones.

"[...] la ruptura de las condiciones de posibilidad de la comprensión de hechos de degradación y muerte, la necesidad de hablar, la urgencia de ser escuchado, la emergencia del silencio para preservar la intimidad o el anonimato, el hueco, el vacío, el mismo dolor" (Aranguren Romero, 2008:21) Entonces, ¿cómo se registra lo no-dicho? ¿Qué contiene el diario de campo? ¿Qué y cómo se puede (y no) registrar en este campo? ¿Es posible lograr la escritura sensible, comprometida y responsable con los estándares de rigurosidad científica? ¿Cómo se escriben los análisis en torno a experiencia traumática?

Si bien esta propuesta se desprende de una investigación previa, fue necesaria hacerla para encontrar no respuestas sino preguntas más ajustadas y sobre todo el lugar o la potencialidad que tenemos como disciplina en estos contextos de trabajo en equipo. Las encrucijadas éticas que se plantean aquí formaron parte de los materiales de análisis, son la guía para ajustar las coordenadas analíticas de la propuesta. Nos movimos dentro de estos parámetros, no sólo por la población seleccionada sino por el tipo de delito que se presenta.

\section{(In)conclusiones}

¿La interdisciplina como perspectiva metodológica de intervención? 
El desafío de comunicadoras y comunicadores en investigación

¿Cómo es esa relación cuerpos investigados, cuerpos que investigan? (Morcillo, 2014). En este cara a cara, la pregunta por la ética profesional y el ejercicio responsable aparecen todo el tiempo. ¿Cuáles son los límites éticos de la escucha y la pregunta? Las preguntas por la experiencia habilitan a reflexionar por esta dimensión sensorial-experiencial del trabajo de campo que lejos de escapar a esta encrucijada que se vuelve ética por momentos, busca reflexionar no solo como insumo sino como mecanismo que interpela todo el tiempo.

La interdisciplina como propuesta metodológica estuvo presente desde el primer momento de la investigación. Sabíamos que debíamos dialogar y buscar tensiones en las disciplinas que se dedican al estudio y abordaje del trauma. No es casual que como comunicadoras y comunicadores ya desde nuestros inicios y comienzos hemos dialogado con otras disciplinas: en los planes de estudios, al interior de las cátedras, en los equipos de investigación, en extensión (dónde mi Universidad tiene una trayectoria muy amplia en trazar puentes con la comunidad) en los pasillos de mates y en las mismas asambleas estudiantiles, luego docentes. Entonces, pensar(nos) en interdisciplina no nos es desconocido, pero sí su abordaje, su pensamiento plural y cómo organizarlo. El desafío fue encontrar el recorte y la mirada entre tanta maraña, ¿qué diferencia a un/a comunicador/a de un/a antropólogo/a, de un/a sociólogo/a en el abordaje de este tipo de problemas sociales? Las organizaciones con las que trabajamos tenían sus tradiciones disciplinares y abordajes específicos de la problemática. ¿Cómo organizar toda esta información? No sólo debíamos encontrar aquellas categorías que nos permitiesen organizar y darle forma a este problema que se presentaba trunco e inaccesible, sino también buscar nuestro lugar en estos equipos y colaborar disciplinarmente. Así, los estudios que dieron sustento a esta propuesta fueron los estudios poscoloniales de género y sexualidad y los estudios culturales en comunicación.

Plantear un mapa analítico no fue casual, significó reconocer quienes son nuestros interlocutores, con quiénes discutimos, concordamos, respondemos. Es insertarnos en una trama de sentido reflexiva donde no estamos solas/os en la tarea de pensar al otro, el ejercicio de recorrer los principales planteos implica ejercitar la reflexividad del investigador (que no solo se despliega en campo) al momento del armado y entramado del mapa analítico y político. Para recorrer y para modificarlo.

La tarea fue la construcción del mapeo analítico teniendo en cuenta todas estas lógicas: la legal, los protocolos de intervención tanto internacionales, locales como específicos de la ONG que asistió, la organización de todas estas dinámicas para dar una propuesta de intervención específica. La comunicación vino a funcionar como articuladora de estas disciplinas, el 
engranaje que permitió tener todos los cuidados necesarios y organizar las notas para la propuesta de protocolos ajustados a todos los cuidados y contextos de circulación. Al conocer las lógicas disciplinares y legales, el análisis de lo que busca una instancia judicial para la resolución de estos delitos y la preservación de las víctimas, desde la comunicación pensamos a partir de los puntos "flojos". La misma es aliada para pensar estrategias específicas de intervención, para encontrar los vacíos y contradicciones dentro de estos vericuetos y traccionar desde ahí. El conocimiento de los formatos que son los "legitimados" para dar cuenta del daño provocado constituye un gran aporte a los equipos que se preparan ante cada intervención judicial como pericia. La comunicación se transforma en una disciplina más que viene a legitimar estos relatos venidos en testimonios, muchas veces sobrevivientes de muerte. La inquietud sigue siendo por la legitimidad de estudios como estos, donde la dignidad e integridad de los sujetos está en juego, colándose en(tre) los relatos, siempre mediando la relación con el otro. Sea cual fueran "los cuidados" que podemos llegar a tener desde las ciencias, siempre ejercemos violencia al momento de encarar situaciones dolorosas. Es en la domesticación o la traducción de algo que no puede ser compartido, esa obligación de pasarlo a categorías científicas, disciplinares, allí está el meollo de la cuestión: ¿cómo investigamos sin invadir, sin violentar? ¿Existe tal cosa? Y la eterna pregunta, ¿cuál es el aporte que podemos hacer desde las ciencias sociales para intervenir y preservar a personas que han atravesado esta experiencia?

\section{Notas}

(1) Lucía Maidana era estudiante de la carrera de Comunicación Social de la Universidad Nacional de Misiones. Fue salvajemente golpeada, violada y prendida fuego en su vivienda del barrio El Palomar de la ciudad de Posadas el 6 de abril del 2013. El crimen involucra a varios estudiantes de la misma facultad, "compañeros" que recorrían los mismos pasillos que Lucía y hoy recorren los mismos pasillos que nosotras. Tenía 22 años. Hasta el momento en que estas líneas fueron escritas no hay detenidos en la causa.

(2) Ex docente de la carrera de Comunicación Social, una de las principales impulsoras de la orientación en investigación en la cual me formé en grado. Investigadora de la FHyCS en temas referidos a movimientos sociales, ciudad y comunicación. Invitada también del panel.

(3) La batería de testeos son los argumentos con los que cuenta la terapeuta para dar cuenta del abuso o, dicho mejor, debe dar cuenta de la psiquis de ese niño/a. No se intenta comprobar una violación sino el daño que produjo ciertos comportamientos en el niño/a que podrían llegar a ser un abuso sexual o violación. Determinados elementos plasmados en esos gráficos, además de señalar que le pasa por dentro a ese niño/a o joven van a encauzar una hipótesis del abuso o violación. 
(4) "La función de los profesionales que realizan la evaluación de las sospechas no es diagnosticar ni certificar si los entrevistados dicen la verdad o si mienten. Su tarea consiste, ni más ni menos, en recoger información de un niño o adolescente, de la manera menos coercitiva posible, y determinar si lo que ha dicho puede ser tenido en cuenta con miras a la puesta en marcha de algún tipo de intervención que podría eventualmente complementarse con una investigación judicial" (Intebi, 2011: 179).

(5) La Comisión tenía por objetivo examinar las causas y consecuencias del conflicto armado interno que tuvo lugar entre los 80 y 90 en Perú. De esta manera, Perú se unió a la creciente lista de países que habían implementado comisiones de verdad como medio de transición de un período de conflicto armado y gobierno autoritario hacia la fundación de una democracia procesual.

\section{Bibliografía}

Aranguren Romero, J. P. (2010). De un dolor a un saber: cuerpo, sufrimiento y memoria en los límites de la escritura. Papeles Del CEIC, pp. 1-27. Recuperado de http://papeles.identidadcolectiva.es/index.php/CEIC/article/view/67

Aranguren Romero, J. P. (2008). El investigador ante lo indecible y lo inenarrable. Revista Nómadas, 29, pp. 20-33.

Barrios, R. (2016). Cuando termine de contar, ¿me voy a seguir acordando de lo que pasó? Análisis de la experiencia narrativa del abuso sexual infantil en clave comunicacional. (Tesis de Doctorado en Comunicación). Facultad de Periodismo y Comunicación, Universidad Nacional de La Plata.

Butler, J. (1999). El género en disputa. México: Paidós.

Butler, J. (1997). Sujetos de sexo/género/deseo. En Feminaria, $X(19)$.

Elizalde, S. (2015). En Foro Clase 1A. Curso: Género y Sexualidades: Debates y herramientas para una educación intercultural. Centro Redes. Recuperado de http://cursos.centroredes.org.ar

Elizalde, S. (2013). Dinámicas culturales de configuración sexo-genérica. En Género y Sexualidades: Debates y herramientas para una educación intercultural. Centro Redes. Recuperado de http://cursos.centroredes.org.ar

Elizalde, S. (2008). Debates sobre la experiencia. Un recorrido por la teoría y la praxis feminista. Revista Oficios Terrestres, XIV(23). Facultad de Periodismo y Comunicación Social, Universidad Nacional de La Plata, pp. 18-30.

Intebi, I. (2011). Proteger, reparar, penalizar. Evaluación de las sospechas de abuso sexual infantil. Buenos Aires: Granica. 
Morcillo, S. (2010). ¿Ir de putas? Reflexiones en torno a las dimensiones sexuadas de la investigación. Revista Kula. Antropólogos Del Atlántico Sur, pp. 7-13.

Pechín, J. (2015). Foro Debate con Juan Pechín. Curso: Género y Sexualidades: Debates y herramientas para una educación intercultural. Centro Redes. Recuperado de http://cursos.centroredes.org.ar

Theidon, K. (2006). Género en transición: sentido común, mujeres y guerra. Cuadernos de Antropología Social, 24, pp. 69-92. 